\title{
Non-tuberculous Mycobacterium Disease: Radiologic Manifestation in an Infant presenting with Respiratory Distress
}

\author{
Rupesh Gautam ${ }^{1}$, Maria Isabel Atienza ${ }^{2,3}$, Maika Noda ${ }^{2}$, and Mariaem \\ Andres ${ }^{2,3}$
}

Chitwan Medical College, Bharatpur, Nepal ${ }^{1}$, St. Luke's Medical Center Quezon City ${ }^{2}$, and St. Luke's Medical Center, Global City ${ }^{3}$

Correspondence to: Dr. Rupesh Gautam, Email: rupesh_gautam@ hotmail.com

\begin{abstract}
Non-tuberculous mycobacterium (NTM) comprises distinct group of organisms with lymphadenitis and pulmonary infection as the common manifestation. The diagnosis of pulmonary disease is based on clinical manifestations, radiologic findings and microbiologic culture. The classic NTM infection may be indistinguishable from pulmonary TB. Nonclassic infection has predilection to the middle lobe and lingula unlike tuberculosis which is commonly seen in the upper lobes. The disease may also present as hypersensitivity pneumonitis with ground glass like opacities, centrilobular nodules and air trapping on imaging. The knowledge of imaging manifestations of NTM will aid in timely diagnosis and treatment of the disease.
\end{abstract}

Keywords: Atypical mycobacterium; Mediastinal lymphadenopathy; Pneumonitis. 


\section{INTRODUCTION}

Tuberculosis remains the second most cause of death due to infectious disease after AIDS. Mycobacterium Tuberculosis (TB) accounts for almost 95 percent cases of the disease, where as non-tubercular mycobacterium (NTM) also known as atypical mycobacterium or Mycobacterium other than Tuberculosis (MOTT) accounts for the remaining 5 percent of disease burden(1).

NTM is a distinct group of organisms with more than 150 species being cataloged and are associated with pulmonary infections, lymphadenitis, skin and soft tissue infections (2). Lymphadenitis is the most common manifestation of NTM infection in the pediatric population, followed by pulmonary disease (3). This case report will try to briefly discuss about NonTubercular Mycobacterial Infection and identify the key imaging features to distinguish it from mycobacterial tuberculosis infection.

\section{Case report}

A 13-week old female infant was admitted to a tertiary medical center in the Philippines for rapid breathing and notable weight loss. The birth history was unremarkable.

Initial chest X-ray showed consolidation at the right upper and both lower lobes. Lateral view demonstrated lobulated soft tissue densities reflective of hilar lymphadenopathy.

Physical examination on admission showed a patient in moderate respiratory distress. I.V antibiotic with Cefuroxime injection was started. On the $3^{\text {rd }}$ hospital day, the patient showed no appreciable improvement. A repeat chest $\mathrm{x}$-ray was then done which revealed progression in extent and degree of the pulmonary disease.

Workup for tuberculosis was pursued. Gastric aspirates for AFB was positive. Anti-TB medications were then given.

Chest CT was performed which revealed bibasal consolidating pneumonia and multiple hilar lymphadenopathy. Necrotizing pneumonia was also observed at the left lung base. Repeat gastric aspirate was taken and sent for culture which eventually revealed Mycobacterium abscessus. The anti-TB medications were discontinued, and the patient was shifted to Amikacin and Clarithromycin.

After almost two months of hospital confinement, patient became stable, afebrile, with good weight gain. She was discharged from the hospital with maintenance IV medications to be given at home. Repeat chest CT demonstrated marked improvement in the pulmonary parenchymal and mediastinal/hilar lymphadenopathy. 
(a)

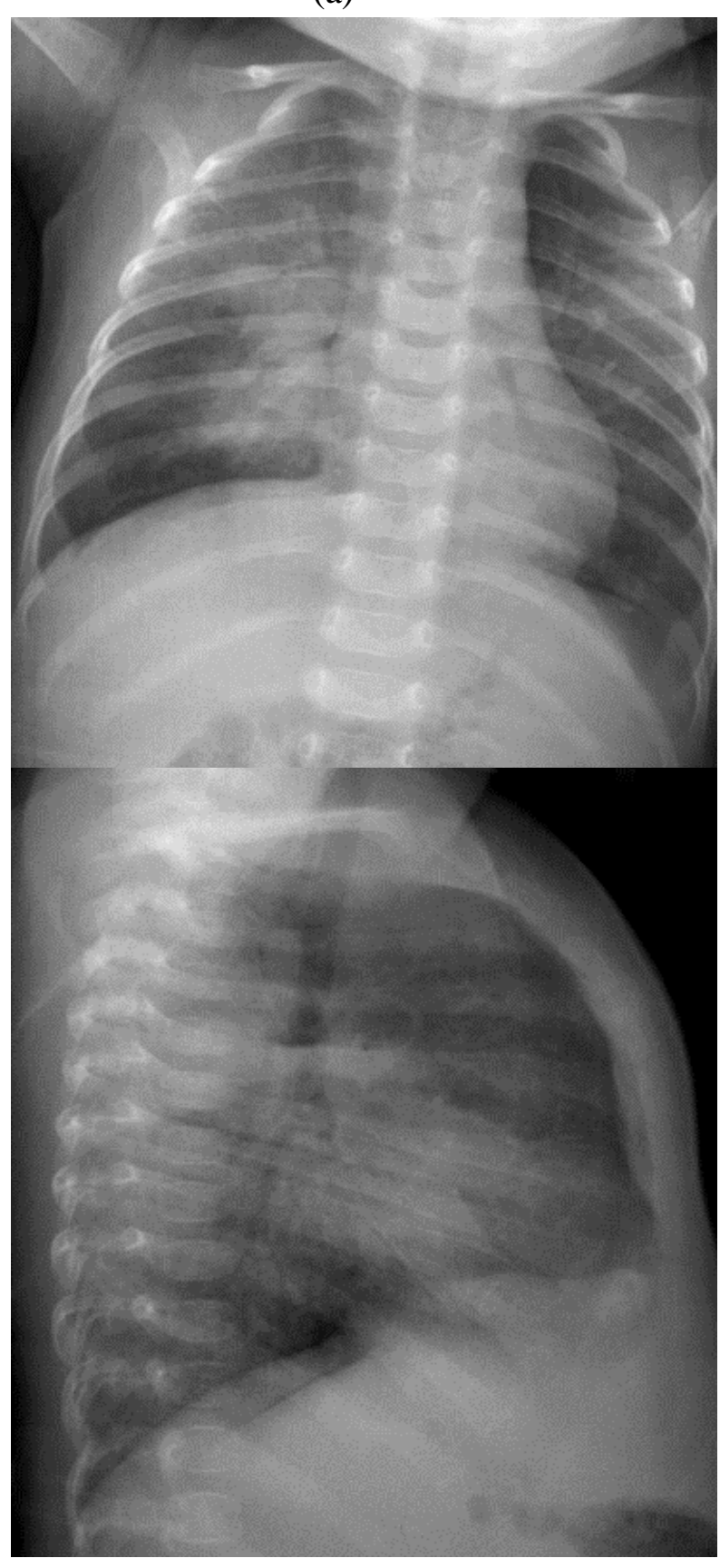

(b)

Figure 1: Chest AP and Lateral view on admission showing consolidation of the right upper and both lower lobes (a). Lateral view (b) showed lobulated soft tissue densities suggestive of hilar lymphadenopathy. (a)

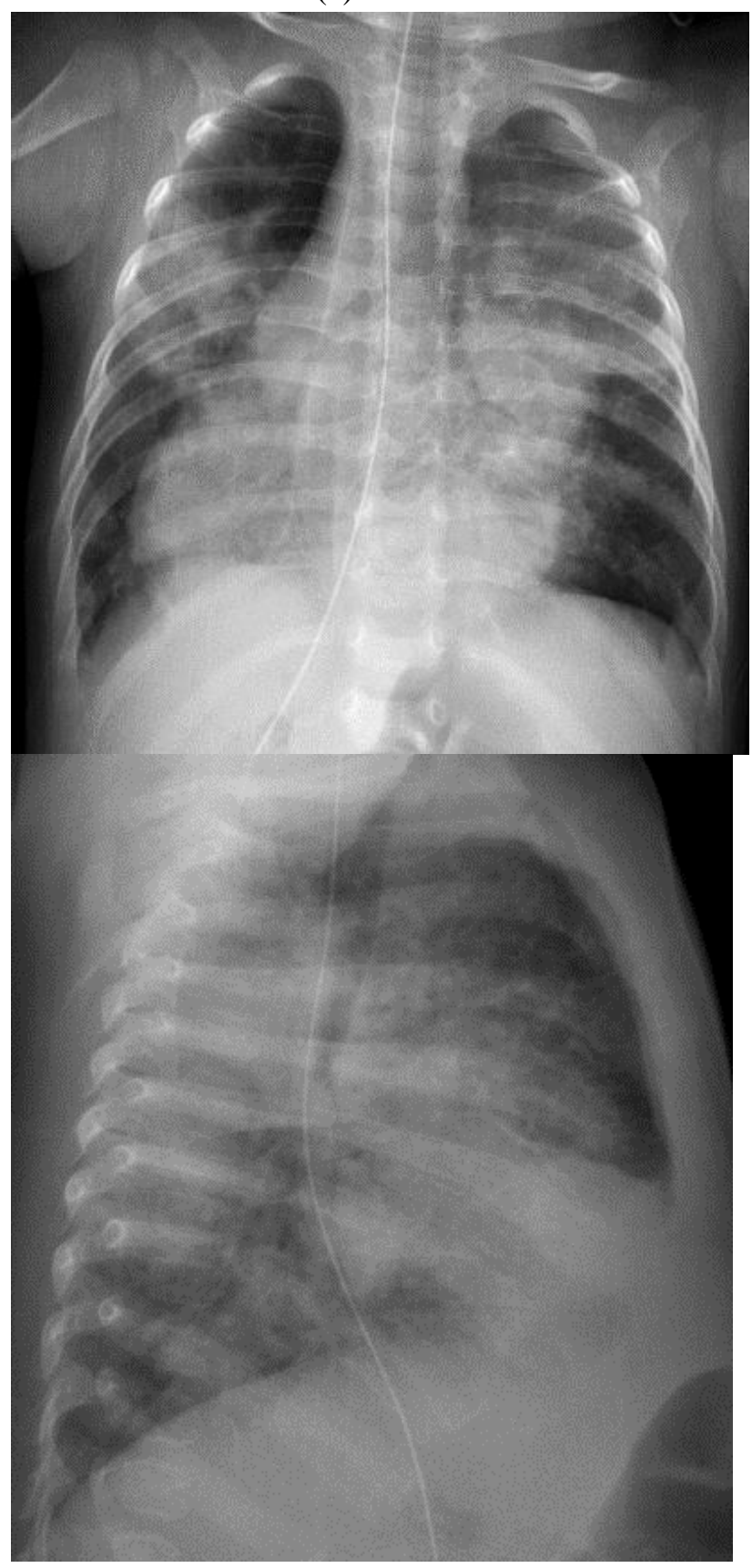

(b)

Figure 2: Follow up chest $\mathrm{x}$-ray shows progression in extent and degree of consolidation at the bilateral upper and both lower lobes in AP view (a). Lateral view (b) again demonstrates lobulated soft tissue densities reflective of hilar lymphadenopathy. 


\section{Gautam R: NON-TUBERCULOUS MYCOBACTERIUM DISEASE}

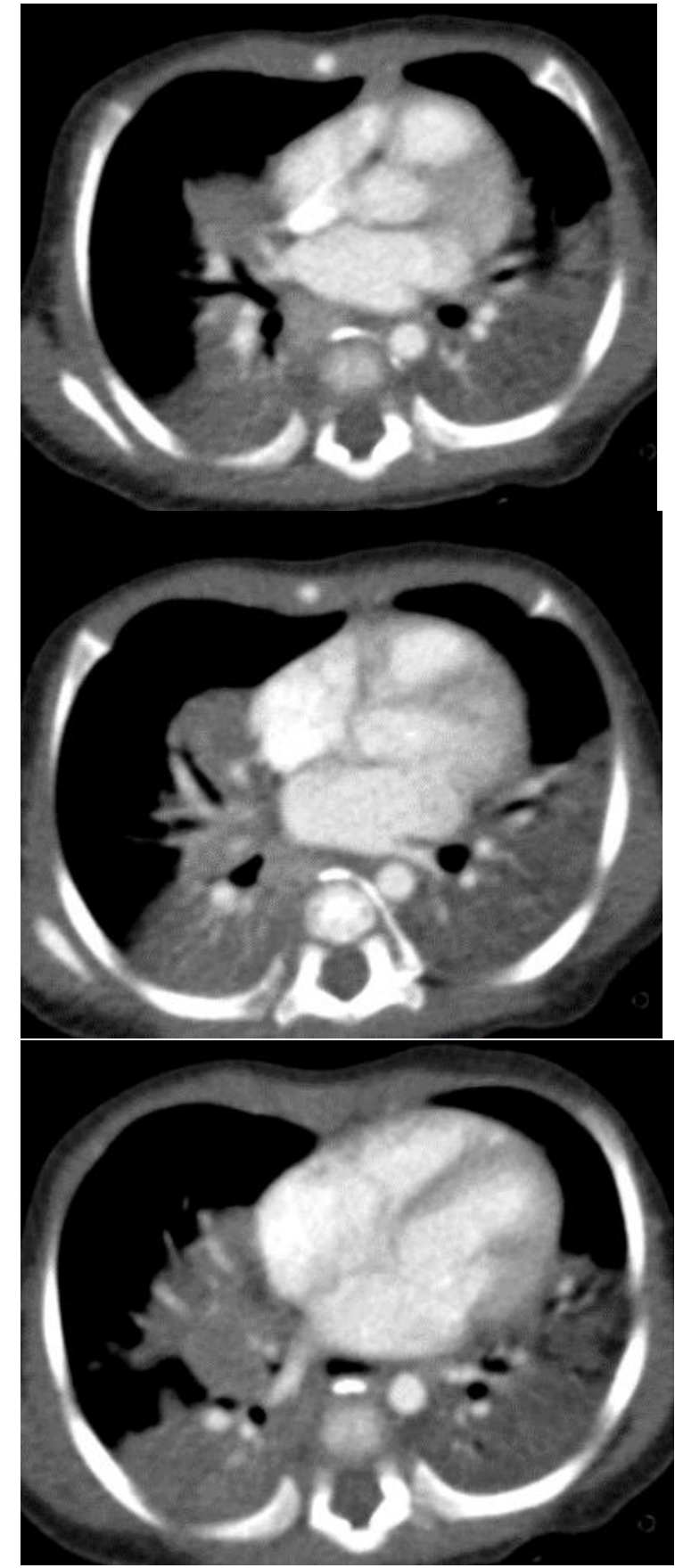

Figure 3: Initial $\mathrm{CT}$ of the chest with intravenous contrast using mediastinal window show series of axial images the demonstrate bibasal lung consolidation, hilar lymphadenopathy, and areas of necrotizing pneumonia at the left lung base.

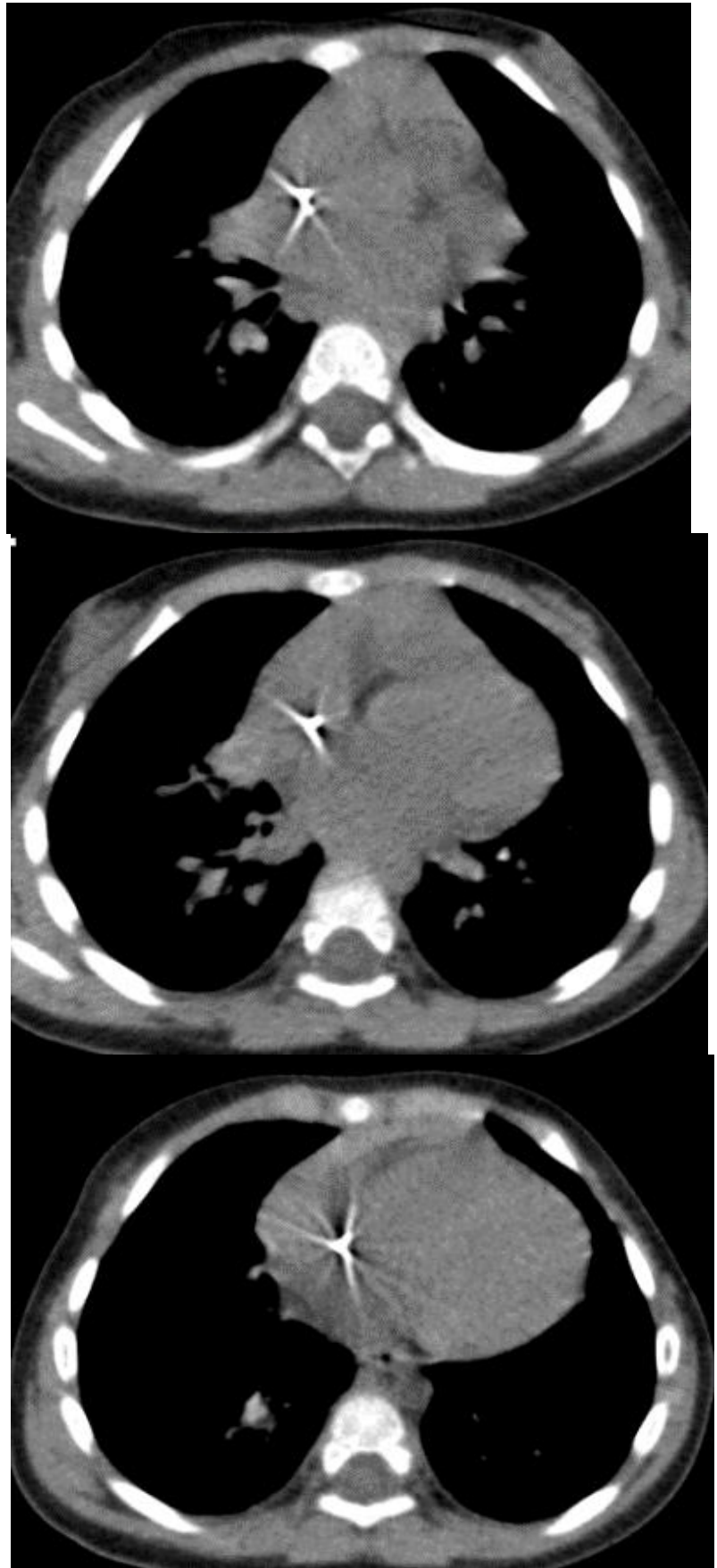

Figure 4: Follow up plain axial chest $\mathrm{CT}$ in mediastinal window show resolution of the parenchymal consolidation, decrease in size of the hilar lymphadenopathy, and resolution of the necrotizing pneumonia on the left lung base. 


\section{DISCUSSION}

NTM are ubiquitous organisms found in soil, water and air. There is no evidence of person-to person transmission; hence isolation of the diseased individual is not required. The major mode of transmission for NTM is presumed to be by droplet inhalation. The most common NTM isolated is Mycobacterium Avium complex (MAC), a slow growing organism. Mycobacterium abscessus is among the most common isolated rapidly growing organism and the third most common isolated NTM species causing lung disease. The other common NTM species are M.Kansasii, M.chelonaeandM.fortuitum (5).

Lymphadenitis as mentioned is the most common manifestation among the pediatric population, where imaging has no significant role and is confirmed by histopathologic examination. Pulmonary manifestation is more common in the elderly population. The American Thoracic Society (ATS) and the infectious Disease Society of America (IDSA) published a joint statement to establish uniform diagnostic criteria for NTM pulmonary disease (Table 1). NMT demonstrates indolent course and usually the clinical symptoms are mild and nonspecific such as chronic cough with sputum and fatigue. Fever and sweats are less frequent than mycobacterium tuberculosis (TB) infection. Weight loss, wasting and hemoptysis are uncommon and if present likely represents advanced disease (4). The disease is confirmed by culture growth of NTM from one bronchoalveolar lavage, two sputum samples or culture from respiratory tissue demonstrating histopathology.

Table 1: American Thoracic Society diagnostic criteria on non tuberculous mycobacterial pulmonary disease

\begin{tabular}{|l|l|}
\hline CT of Tuberculous & Mycobacterial \\
Infection & CT of Non-tuberculous Mycobacterial Infection \\
\hline Thick walled cavity $(\mathbf{3} \mathbf{~ c m ) ~}$ & Thin walled cavity $(<\mathbf{3} \mathbf{c m})$ \\
\hline Pulmonary consolidation & Small parenchymal nodules \\
\hline Patchy and reticular infiltrates & Lymph node calcification \\
\hline $\begin{array}{l}\text { Atelectasis } \\
\text { Mediastinal and hilar lymph nodes }\end{array}$ & \\
\hline $\begin{array}{l}\text { Lymph node calcification } \\
\text { Pleural effusion }\end{array}$ & \\
\hline
\end{tabular}

Chest $\mathrm{x}$-ray is the primary imaging modality that describes the parenchymal and lymph node involvement in a patient affected with tuberculosis. Further evaluation can be performed with a chest CT scan to elucidate the details of the bronchial lumina whether luminal compromise or bronchiectasis is present, the presence of nodules, consolidation, atelectasis, cavity, or possibly necrotic changes in mediastinal and hilar lymph nodes. 
Miller classified pulmonary NTM infection according to its clinical presentation and imaging manifestations as 1) classical (cavitary), 2) non-classical (bronchiectatic), 3) associated with immunosupression, 4) achalasia like symptoms and 5) nodules or masses in asymptomatic individuals (6). Tuberculosis and non-tubercular mycobacterial disease have similar imaging features and may be indistinguishable solely on the basis of imaging manifestations.

Classic infection is the most common form of NTM disease with imaging features similar to post primary pulmonary tuberculosis. The radiological features are cavitations, ill defined linear and nodular opacities predominantly at the upper lobes. These are better characterized with CT scan. Endobronchial spread of the NTM disease from the cavities are common and results in tree in bud like centrilobular nodules adjacent to the disease foci. Bronchiectatic and cicatricial atelectasis are also seen at the upper lobes.

The lower lobe involvement is uncommon.CT scan demonstrates cavities which are usually smaller and thin walled than tuberculosis. The mean size of the cavities are less than $2.5 \mathrm{~cm}$.Likewise pleural effusion, calcified pulmonary nodules and mediastinal lymph nodes ( Ranke's complex ) are less common than in post primary pulmonary tuberculosis. $(7,8)$ The disease process demonstrates relatively slower course than active pulmonary tuberculosis. The involvement of pleura as pleural thickening adjacent to the parenchymal disease is more pronounced in NTM disease as compared to tuberculosis; however basal pleural disease is rare unlike tuberculosis. Mycobacterium Avium Complex (MAC) is the most common organism responsible for this form of disease and commonly seen in elderly patients with preexisting lung disease like COPD.

Non-classic infection or bronchiectatic form of NTM disease is seen usually in elderly women without history of coexisting lung disease. Chronic cough may be the only clinical manifestation in majority of the patients.

Lady Windermere syndrome is associated with non mycobacterial tubercular disease where there is voluntary cough suppression, leading to aspiration and engraftment of MAC infected secretions. The imaging features of non classic form of NTM disease are usually symmetric and cylindrical bronchiectasis, more common in the lingula and middle lobe and bronchiolitis in the form of tree in bud like nodules. Atelectasis, cavitations and mediastinal lymphadenopathy are uncommon $(7,8)$. The more common involvement of middle lobe and lingula is may help in the diagnosis of the disease which is unlikely for mycobacterium tuberculosis (TB).

Non-tubercular mycobacterial disease is seen with immunosuppression state in both HIV and non-HIV patients. The disease manifests in HIV patients with low CD4 counts (less than 100 cells $/ \mathrm{mm}^{5}$ ) and are usually associated coexisting infections like pneumocystis, cytomegalovirus, and fungal infection (7). This overlapping disease makes difficult to identify the only imaging features NTM disease in this group of patients. MAC is 
the most common NTM organism associated with HIV infection and usually presents with disseminated disease. The pertinent imaging feature is mediastinal lymphadenopathy and less likely miliary nodules, pleural effusion and air space opacities. The other organisms associated with HIV infections are M.Kansasii and M.Xenopi with imaging features similar to disseminated Tubercular disease (TB).Non HIV group of immunosuppressed patients includes the patients on chemotherapy, organ transplantation, leukemia, lymphoma and many others.MAC and M.Kansasii are again the most common organisms for the disease and usually presents with constitutional symptoms like fever, malaise, weight loss, cough. The imaging features are reticulonodular opacities, mediastinal and hilar lymphadenopathies with no significant features to distinguish it from Mycobacterium Tuberculosis $(7,8)$.

NTM has been also reported to be associated with deglutition disorders like achalasia, stroke, Parkinsonism and others. $M$. fortuitumand $M$. chelonaeare the organisms isolated. The imaging manifestations are either reticulo nodular or air space filling opacities like aspiration pneumonia with no definite lobar predominance (8). Pulmonary NTM may manifest as nodules or masses in asymptomatic individuals.MAC are the most common isolated organism. The nodules are usually of uniform size clustered together. Some of these nodules may manifest with internal calcifications or hyper densities, reflective of granulomatous disease $(7,8)$.

There are few reported cases of hypersensitivity pneumonitis secondary to
NTM disease. These organisms were also isolated from house hold waters and may manifests as hot tub lung disease. Imaging manifestations are ill-defined, groundglass centrilobular nodules, diffuse ground-glass opacities, consolidations and air trapping on expiratory examination (7).

\section{CONCLUSION}

Pulmonary NTM disease is clinically and radiographically indistinguishable from pulmonary tuberculosis and therefore might not be considered in children assessed for pulmonary tuberculosis.

\section{REFERENCES}

1. Kahkouee S, Esmi E, Moghadam A, Karam MB, Mosadegh L, Salek S, Tabarsi $\mathrm{P}$ et al. Multidrug resistant tuberculosis versus non-tuberculous mycobacterial infections: a CT-scan challenge. Braz J Infect Dis 2013; 17(2):

137-142.

2. Johnson MM, Odel JA. Nontuberculous mycobacterial pulmonary infections. $J$ Thorac Dis 2014; 6(3): 210-22.

3. Haverkamp MH, Arend SM, Lindeboom JA, Hartwig NG, Dissel JT. Nontuberculous Mycobacterial Infection in Children: A 2-Year Prospective Surveillance Study in the Netherlands. Clinical Infectious Diseases 2004; 39 (4): 450-456.

4. Glassroth J. Pulmonary Disease Due to Nontuberculous Mycobacteria. CHEST 2008; 133(1):243-251.

5. Griffith DE, Aksamit T, Brown-Elliott BA, Catanzaro A, Daley C, Gordin F et al. An Official ATS/IDSA Statement: Diagnosis, Treatment, and 
prevention of Nontuberculous Mycobacterial Diseases. Am J Respir Crit Care Med 2007; 175(4): 367-417.

6. Miller WT. Spectrum of Pulmonary nontubercular mycobacterial infection. Radiology 1994; $191(2)$ : 343-350.

7. Martinez S, McAdams PH, Batchu CS. The Many Faces of Pulmonary Nontuberculous Mycobacterial Infection. AJR 2007; 189(1):177-186.

8. Erasmus JJ, McAdams HP, Farrell MA, Patz EF. Pulmonary Nontuberculous Mycobacterial infection: Radiological Manifestations. Radiographics 1999; 19 (6):1487-1503. 